\title{
Long-term effectiveness of agalsidase alfa enzyme replacement in Fabry disease: A Fabry Outcome Survey analysis
}

\author{
Michael Beck ${ }^{\mathrm{a}, *}$, Derralynn Hughes ${ }^{\mathrm{b}}$, Christoph Kampmann ${ }^{\mathrm{a}}$, Sylvain Larroque ${ }^{\mathrm{c}}$, Atul Mehta ${ }^{\mathrm{b}}$, \\ Guillem Pintos-Morell ${ }^{\mathrm{d}}$, Uma Ramaswami ${ }^{\mathrm{b}}$, Michael West ${ }^{\mathrm{e}}$, Anna Wijatyk ${ }^{\mathrm{f}}$, Roberto Giugliani ${ }^{\mathrm{g}}$, \\ the Fabry Outcome Survey Study Group
}

\footnotetext{
a University Medical Center, University of Mainz, Department of Paediatrics, Mainz, Germany

${ }^{\mathrm{b}}$ Royal Free London NHS Foundation Trust, University College of London, UK

c Shire, Zug, Switzerland

d Department of Pediatrics, University Hospital “Germans Trias i Pujol," Badalona, Universitat Autònoma de Barcelona, Spain

e Department of Medicine, Dalhousie University, Halifax, Nova Scotia, Canada

f Shire, Lexington, MA, USA

${ }^{g}$ Medical Genetics Service HCPA/Dep Genet UFRGS and INAGEMP, Porto Alegre, Brazil
}

\section{A R T I C L E I N F O}

\section{Article history:}

Received 12 February 2015

Accepted 12 February 2015

Available online 5 March 2015

\section{Keywords:}

Fabry disease

Enzyme replacement therapy

Agalsidase alfa

Long-term effectiveness

\begin{abstract}
A B S T R A C T
Outcomes from 5 years of treatment with agalsidase alfa enzyme replacement therapy (ERT) for Fabry disease in patients enrolled in the Fabry Outcome Survey (FOS) were compared with published findings for untreated patients with Fabry disease. Data were extracted from FOS, a Shire-sponsored database, for comparison with data from three published studies. Outcomes evaluated were the annualized rate of change in estimated glomerular filtration rate (eGFR) and left ventricular mass indexed to height (LVMI) as well as time to and ages at a composite morbidity endpoint and at death. FOS data were extracted for 740 treated patients who were followed for a median of $\sim 5$ years. Compared with no treatment, patients treated with agalsidase alfa demonstrated slower decline in renal function and slower progression of left ventricular hypertrophy. Treated male patients with baseline eGFR $<60 \mathrm{~mL} / \mathrm{min} / 1.73 \mathrm{~m}^{2}$ had a mean (standard error of the mean [SEM]) annualized change in eGFR of $-2.86(0.53) \mathrm{mL} / \mathrm{min} / 1.73 \mathrm{~m}^{2} / \mathrm{y}$ compared with $-6.8(1.5)$ in the published untreated cohort. The mean (SEM) rate of LVMI increase with treatment was $0.33(0.10) \mathrm{g} / \mathrm{m}^{2.7} / \mathrm{y}$ in males and $0.48(0.09)$ in females, compared with 4.07 (1.03) in untreated males and 2.31 (0.81) in untreated females. Morbidity occurred later in treated patients, with 16\% risk of a composite morbidity event ( $26 \%$ in males) after 24 months with ERT versus 45\% without treatment, with first events and deaths also occurring at older ages in patients administered ERT (e.g., estimated median survival in treated males was 77.5 years versus 60 years in untreated males). Findings from these retrospective comparisons of observational data and published literature support the long-term benefits of ERT with agalsidase alfa for Fabry disease in slowing the progression of renal impairment and cardiomyopathy. Treatment also appeared to delay the onset of morbidity and mortality. Interpretation of these findings should take into account that they are based on retrospective comparisons with previously published data.

(C) 2015 The Authors. Published by Elsevier Inc. This is an open access article under the CC BY-NC-ND license
\end{abstract} (http://creativecommons.org/licenses/by-nc-nd/4.0/).

\section{Introduction}

Fabry disease (OMIM 301500) is a rare inherited X-linked glycosphingolipid storage disorder in which mutations in the $\alpha$-galactosidase A gene result in functional deficiency of the lysosomal

Abbreviations: ACEI, Angiotensin-converting enzyme inhibitor; ARB, Angiotensin receptor blocker; CI, Confidence interval; eGFR, Estimated glomerular filtration rate; ERT, Enzyme replacement therapy; FOS, Fabry Outcome Survey; LVH, Left ventricular hypertrophy; LVMI Left ventricular mass indexed to height; MDRD, Modification of Diet in Renal Disease; SE, Standard error; SEM, Standard error of the mean.

* Corresponding author at: Children's Hospital, University Medical Centre, J.G. University Mainz, Langenbeckstrasse 1, 55101 Mainz, Germany.

E-mail address: Michael.Beck@unimedizin-mainz.de (M. Beck). enzyme $\alpha$-galactosidase (EC 3.2.1.22) [1]. This leads to progressive accumulation of glycosphingolipids, particularly globotriaosylceramide, in almost all tissues and organs. The most serious complications in adult patients are progressive renal impairment, cardiomyopathy, and cerebrovascular events, all of which lead to significant morbidity and mortality and reduced life expectancy [2-8].

Enzyme replacement therapy (ERT) for Fabry disease has been available for $>10$ years [9-13]. Currently, it is the only approved approach to disease modification, and patients are likely to be on long-term treatment. The international Fabry Outcome Survey (FOS), sponsored by Shire (Lexington, MA, USA), was initiated in 2001 to collect long-term clinical and safety outcomes data for individuals with confirmed diagnosis of Fabry disease who either receive agalsidase 
alfa treatment or no ERT [14]. This ongoing database has facilitated the study of many aspects of Fabry disease and the effects of ERT, including renal and cardiac outcomes [2,11,15-19].

A past limitation of FOS has been the lack of a robust comparable untreated cohort. The untreated population in FOS is generally less severely affected than patients receiving treatment and includes a higher proportion of females. Untreated patients also have tended to undergo fewer follow-up assessments and therefore have less outcome data available [11]. An exploratory analysis suggested that $<10 \%$ of FOS participants could be matched for comparison. Longterm data on mortality, morbidity, and progression of renal impairment and cardiac disease without ERT are, however, available from other published studies [8,20].

The objective of our analysis was to compare the long-term renal and cardiac consequences and morbidity and mortality outcomes for agalsidase alfa-treated patients from FOS with well-described cohorts of untreated individuals from previously published studies.

\section{Methods}

\subsection{Patients}

Patients included in FOS are either receiving agalsidase alfa (Replagal ${ }^{\circledR}$; Shire, Lexington, MA, USA) at the approved dosage or are not receiving any ERT. Details of data collection have been presented elsewhere $[11,14,15,19]$. Patients who had been treated with another ERT before FOS entry were excluded from this analysis. Patients who left FOS to be treated with another ERT were censored from the analyses from the date of leaving. For this analysis, FOS data were extracted for the period from database inception in 2001 through November 2012. FOS has been approved by the ethics institutional review boards of participating centers. All participants gave written informed consent.
Data for untreated patients were derived from three published sources (Fig. 1) [8,20,21]. Mortality, morbidity, and the progression of renal impairment were explored in a multinational Fabry disease retrospective chart study by Schiffmann et al. [21]. Cardiac changes over time were examined in a two-center prospective, cross-sectional, longitudinal study by Kampmann et al. [8]. Time from baseline to onset of first clinical event (e.g., death, cardiac, renal, or cerebrovascular) was evaluated in a multinational randomized clinical trial of agalsidase beta versus placebo by Banikazemi et al.; the placebo arm of this study was used as an untreated comparator cohort [20].

Five different patient populations from FOS were defined to provide comparator populations that were as closely matched as possible to the published untreated cohorts (Fig. 1). The All Treated cohort comprised all patients (children and adults) from FOS who started agalsidase alfa either before or during FOS entry. The Evaluable Treated cohort, a subset of the All Treated cohort, excluded patients who had undergone renal transplant or renal dialysis prior to treatment. Three further subsets of the Evaluable Treated cohort were also defined. The Evaluable Treated Morbidity cohort for comparison with data from Banikazemi et al. [20] comprised adult patients who had a baseline estimated glomerular filtration rate $(\mathrm{eGFR})<80 \mathrm{~mL} / \mathrm{min} / 1.73 \mathrm{~m}^{2}$ at treatment start. The Evaluable Treated Renal cohort for comparison with untreated patients from Schiffmann et al. [21] included those adults who had $\geq 3$ eGFR Modification of Diet in Renal Disease (MDRD) values, including at baseline and 2 years. An Evaluable Treated Cardiac cohort for evaluation against data from Kampmann et al. [8] included patients with $\geq 3$ left ventricular mass indexed to height (LVMI) measurements, including at baseline and 2 years.

\subsection{Outcomes analyzed}

The rate of change in renal function was evaluated as the annualized change in eGFR in adults (aged $\geq 18$ years), using a four-variable MDRD eGFR equation [22]. Cardiomyopathy was similarly determined

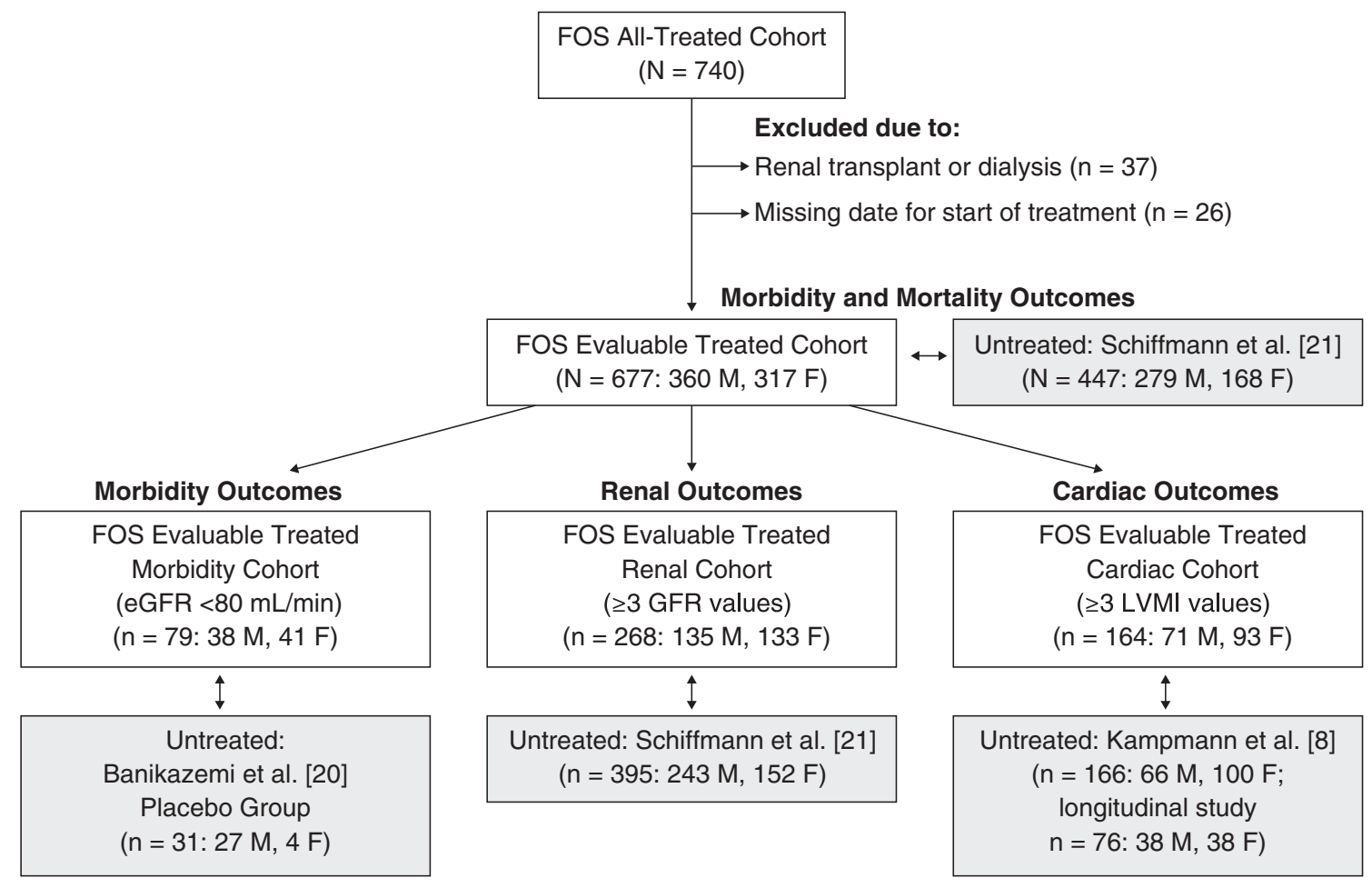

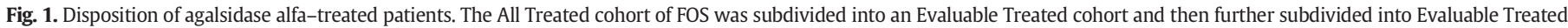

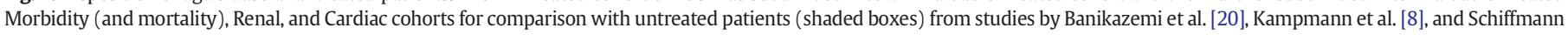
et al. [21]. eGFR, estimated glomerular filtration rate; F, female; FOS, Fabry Outcome Survey; LVMI, left ventricular mass indexed to height; M, male. 
from annualized slopes in LVMI, calculated from echocardiogram findings [23].

Morbidity, as time to and age at first event, was determined using two clinical composite event endpoints. To compare with untreated patients from Banikazemi et al. [20], the endpoint comprised myocardial infarction, any serious cardiac event indicative of coronary artery disease, heart failure, valvular disease, or arrhythmia; any event resulting in or reported as percutaneous transluminal coronary angioplasty or coronary artery bypass graft; any serious heart failure event; serious events requiring valvular surgery; serious adverse events stating or indicating renal transplant, dialysis, or indicative of chronic dialysis; an increase in serum creatinine by $33 \%$ from baseline (two consecutive values); cerebrovascular accident, stroke or transient ischemic attack, or death. The second composite of any myocardial infarction, cardiac surgery, arrhythmia, angina, or heart failure; renal transplant or dialysis, initiation of renal transplant or dialysis or increase in serum creatinine by $33 \%$ from baseline (two consecutive values); stroke, transient ischemic attack, or prolonged reversible ischemic neurologic disorder; and/or death was used for comparison with results by Schiffmann et al. [21]. Mortality in FOS, evaluated from fatality forms, adverse events forms, and exit forms, was also compared with the data from Schiffmann et al. [21].

\subsection{Statistical analyses}

Progression of renal disease and cardiomyopathy was calculated from average slopes from mixed models of available eGFR and LVMI data, using the following equations:

$$
\begin{aligned}
M D R D= & \text { Intercept }+\alpha . \text { Age }+\beta \text {.Gender }+\chi . \text { Baseline } M D R D+\delta . \text { Age } \\
& \times \text { Gender }+\eta \text {.Age } \times \text { Baseline } M D R D+\mu . \text { Gender } \\
& \times \text { Baseline } M D R D+\theta . \text { Age } \times \text { Gender } \times \text { Baseline } M D R D+\varepsilon
\end{aligned}
$$

$$
\begin{aligned}
\mathrm{LVMI}= & \text { Intercept }+\alpha . \text { Age }+\beta . \text { Gender }+\chi \text {. Baseline } \mathrm{LVH}+\delta . \text { Age } \\
& \times \text { Gender }+\eta \text {.Age } \times \text { Baseline } \mathrm{LVH}+\mu \text {.Gender } \times \text { Baseline } \mathrm{LVH} \\
& +\theta . \text { Age } \times \text { Gender } \times \text { Baseline } \mathrm{LVH}+\varepsilon .
\end{aligned}
$$

The interaction model procedure PROCMIXED in SAS/STAT ${ }^{\circledR}$ version 9.2 (SAS Institute Inc., Cary, NC, USA) was used to derive eGFR and LVMI rate of change estimates $r$. For eGFR evaluations, values after dialysis or transplantation were removed from the model estimations; serum creatinine values $<0.2 \mathrm{mg} / \mathrm{dL}$ or $>15 \mathrm{mg} / \mathrm{dL}$ were also excluded. For LVMI analyses, LVMI values $<5 \mathrm{~g} / \mathrm{m}^{2.7}$ or $>1000 \mathrm{~g} / \mathrm{m}^{2.7}$ were excluded. Confidence intervals (CIs) were calculated for FOS data; however, corresponding Cls were not available for many of the published data.

Kaplan-Meier time to morbidity analyses were conducted for FOS participants, running from treatment start to the first occurrence of composite event (for events on or after treatment start only), with censoring for the first of either at last visit or 35 months. Kaplan-Meier analyses of FOS data were also conducted for age at morbidity, running from birth to first composite event, and for mortality, with censoring at last visit (or date of leaving for patients who left FOS to be treated with another ERT).

\subsection{Sensitivity analyses}

Each pair of cohorts was compared descriptively to identify differences in baseline clinical and demographic parameters. Where such differences were found, confirmatory analysis of randomly resampled subgroups of the population in question was conducted.

Where significant differences in outcomes were detected in the study, supportive subgroup analyses were conducted to evaluate sensitivity and robustness. For cardiac and renal outcomes, analyses were repeated for treated patients who had available 5-year follow-up data. An additional sensitivity analysis was done with treated patients who had renal transplant or dialysis before FOS entry. For mortality, a supportive analysis for the All Treated cohort was performed, and results were confirmed by random resampling to match the published untreated population. For morbidity, supportive analyses for the endpoints from Schiffmann et al. [21] and Banikazemi et al. [20] were conducted for the first occurrence of events after adding events from FOS signs and symptoms forms that had not already been captured as serious adverse events. The original FOS analysis included patients with transient ischemic attack, stroke, or myocardial infarction during the 3 months prior to initiating ERT, whereas such patients were excluded from the untreated Banikazemi et al. cohort.

\section{Results and discussion}

At data extraction (November 15, 2012), the FOS All Treated cohort comprised 740 patients from expert sites in 19 countries. Treated patient cohorts for analyses were derived from this population (Fig. 1). We have retrospectively examined long-term changes in renal function, cardiomyopathy, morbidity, and mortality in patients with Fabry disease who had been treated with agalsidase alfa and compared these results with similar cohorts of untreated patients from published studies. This approach was taken because an effective comparison of treated and untreated patients from FOS was not feasible. As already noted, untreated patients in FOS tended to have less severe disease; also, less clinical data have been collected (e.g., safety reporting requirements did not apply in the same manner to untreated patients [11,14]). Deriving matched treated and untreated cohorts from FOS would therefore have generated comparator groups too small to yield valid conclusions. Two of the untreated cohorts utilized for this study were from natural history studies [8,21], and the third was the placebo group in a randomized trial of agalsidase beta [20]. Data from FOS were extracted to provide cohorts of treated patients corresponding as closely as possible to those from the published studies in terms of inclusion/exclusion criteria and data available.

The FOS Evaluable Treated Renal cohort and corresponding untreated patients from Schiffmann et al. [21] were broadly comparable in age and renal function characteristics (Table 1). The FOS Evaluable Cardiac cohort and the comparator Kampmann et al. [8] cohort were closely matched in terms of numbers of patients, age, and relevant clinical factors (Table 2). Of the two untreated cohorts available from the literature for evaluation of morbidity-related outcomes, the cohort from Banikazemi et al. [20] comprised mostly males ( $87 \%$ versus $48 \%$ in the FOS Evaluable Morbidity cohort; Table 3). The untreated cohort from Schiffmann et al. [21] was similar to the gender balance in the matched FOS cohort (Table 3).

Over a median follow-up of $\sim 5$ years (median [range] interval between first and last eGFR assessment, FOS 5.4 [1.5 to 13.7] years), the estimated annualized eGFR change with treatment was -2.86 (standard error of the mean [SEM] 0.53) $\mathrm{mL} / \mathrm{min} / 1.73 \mathrm{~m}^{2} / \mathrm{y}$ among male patients with poor renal function at baseline (eGFR $<60 \mathrm{~mL} / \mathrm{min} / 1.73 \mathrm{~m}^{2}$; Table 4). In patients with higher baseline eGFR $\left(\geq 60 \mathrm{~mL} / \mathrm{min} / 1.73 \mathrm{~m}^{2}\right)$, the annualized rate of decline in eGFR was less than in patients with lower baseline eGFR (Table 4). In comparison, the untreated cohort from Schiffmann et al. [21], with a median (range) followup (interval between first and last eGFR assessment) of 5.6 [0.1 to 28.2] years, had an estimated annualized eGFR change of -6.8 (SEM 1.5 ) $\mathrm{mL} / \mathrm{min} / 1.73 \mathrm{~m}^{2} / \mathrm{y}$. When comparing the FOS Evaluable Treated Renal cohort and the Schiffmann et al. untreated cohort, the effect of ERT on annualized eGFR change was seen across all baseline urinary protein level categories (Table 4). In a sensitivity analysis, eGFR results were evaluated only for FOS ERT patients who had 5-year data (79 males, 71 females; median [range] treatment duration, 8.25 [2.7 to 13.8 ] years). The annualized eGFR slopes for these patients, overall and grouped by baseline eGFR, were not significantly different from 
Table 1

Baseline renal function-related clinical characteristics of patients from FOS and Schiffmann et al. [21] who were included in the analyses of the progression of renal impairment.

\begin{tabular}{|c|c|c|c|c|c|c|c|c|}
\hline \multirow{4}{*}{ Parameter (mean at baseline) } & \multicolumn{8}{|c|}{ By baseline eGFR $\left(\mathrm{mL} / \mathrm{min} / 1.73 \mathrm{~m}^{2}\right)$} \\
\hline & \multicolumn{4}{|c|}{ FOS Evaluable Treated Renal cohort } & \multicolumn{4}{|c|}{ Schiffmann et al. (untreated) [21] } \\
\hline & \multicolumn{2}{|c|}{ Males $(n=135)$} & \multicolumn{2}{|c|}{ Females $(\mathrm{n}=133)$} & \multicolumn{2}{|c|}{ Males $(\mathrm{n}=243)$} & \multicolumn{2}{|c|}{ Females $(\mathrm{n}=152)$} \\
\hline & $\geq 60(n=117)$ & $<60(\mathrm{n}=18)$ & $\geq 60(n=111)$ & $<60(\mathrm{n}=22)$ & $\geq 60(n=189)$ & $<60(\mathrm{n}=54)$ & $\geq 60(\mathrm{n}=129)$ & $<60(\mathrm{n}=23)$ \\
\hline Age (yrs) & 34.7 & 44.1 & 44.1 & 55.8 & 27.3 & 41.8 & 38.0 & 51.9 \\
\hline Urinary protein $(\mathrm{g} / 24 \mathrm{~h})$ & 0.4 & 1.5 & 0.5 & 1.0 & 0.6 & 2.6 & 0.3 & 1.1 \\
\hline Overt proteinuria (\%) & 40 & 93 & 37 & 56 & 37 & 96 & 20 & 50 \\
\hline Systolic BP (mm Hg) & 124 & 124 & 126 & 133 & 125 & 128 & 123 & 135 \\
\hline Diastolic BP (mm Hg) & 73 & 77 & 74 & 76 & 74 & 79 & 75 & 82 \\
\hline
\end{tabular}

(

By baseline urinary protein $(\mathrm{g} / 24 \mathrm{~h})$

Parameter

(mean at baseline)
FOS Evaluable Treated Renal cohort

Males $(\mathrm{n}=105)$

\begin{tabular}{|c|c|c|c|c|c|}
\hline \multicolumn{3}{|c|}{ Males $(\mathrm{n}=105)$} & \multicolumn{3}{|c|}{ Females $(\mathrm{n}=109)$} \\
\hline $\begin{array}{l}\geq 1.0 \\
(n=16)\end{array}$ & $\begin{array}{l}0.1 \text { to } 1.0 \\
(\mathrm{n}=74)\end{array}$ & $\begin{array}{l}<0.1 \\
(n=15)\end{array}$ & $\begin{array}{l}\geq 1.0 \\
(n=17)\end{array}$ & $\begin{array}{l}0.1 \text { to } 1.0 \\
(n=70)\end{array}$ & $\begin{array}{l}<0.1 \\
(n=22)\end{array}$ \\
\hline 38.8 & 36.1 & 37.0 & 46.9 & 45.7 & 42.1 \\
\hline 74.4 & 101.8 & 105.0 & 65.7 & 87.3 & 80.2 \\
\hline
\end{tabular}

\begin{tabular}{|c|c|c|c|c|c|}
\hline \multicolumn{6}{|c|}{ Schiffmann et al. (untreated) [21] } \\
\hline \multicolumn{3}{|c|}{ Males $(\mathrm{n}=61)$} & \multicolumn{3}{|c|}{ Females $(\mathrm{n}=29)$} \\
\hline $\begin{array}{l}\geq 1.0 \\
(n=22)\end{array}$ & $\begin{array}{l}0.1 \text { to } 1.0 \\
(\mathrm{n}=21)\end{array}$ & $\begin{array}{l}<0.1 \\
(n=18)\end{array}$ & $\begin{array}{l}\geq 1.0 \\
(n=5)\end{array}$ & $\begin{array}{l}0.1 \text { to } 1.0 \\
(\mathrm{n}=17)\end{array}$ & $\begin{array}{l}<0.1 \\
(\mathrm{n}=7)\end{array}$ \\
\hline 38.9 & 36.0 & 22.8 & 47.2 & 42.3 & 39.0 \\
\hline 58.5 & 84.6 & 138.0 & 63.4 & 89.6 & 91.9 \\
\hline
\end{tabular}

\begin{tabular}{lccccc} 
Age $(\mathrm{yrs})$ & 38.8 & 36.1 & 37.0 & 46.9 & 45.7 \\
eGFR $\left(\mathrm{mL} / \mathrm{min} / 1.73 \mathrm{~m}^{2}\right)$ & 74.4 & 101.8 & 105.0 & 65.7 & 87.3 \\
\hline BP, blood pressure; eGFR, estimated glomerular filtration rate; & FOS, Fabry Outcome Survey.
\end{tabular}

corresponding values for the full FOS ERT renal cohort. Another sensitivity analysis looked at eGFR results when patients on dialysis or those that had received a renal transplant before FOS entry were included in the FOS Evaluable Treated Renal cohort (Supplemental Tables 1 and 2).

A number of patients in each cohort had been treated with angiotensin-converting enzyme inhibitors (ACEI) or angiotensin receptor blockers (ARB), which is a potential source of bias. In the untreated cohort, $20 \%(n=48)$ of males and $12 \%(n=18)$ of females had received ACEI [21], compared with $57 \%(n=77)$ of males and $65 \%(n=87)$ of females in the FOS Evaluable Treated Renal cohort who had received either ACEI or ARB at some time during the periods analyzed. In treated patients, the annualized slopes of eGFR were not significantly different between patients who had or had not received an ACEI or ARB $(-1.37$ [SEM 0.22; 95\% CI $-1.81,-0.94 ; \mathrm{n}=164] \mathrm{mL} / \mathrm{min} / 1.73 \mathrm{~m}^{2} / \mathrm{y}$ and -1.48 [SEM 0.84; 95\% CI $-3.15,0.19 ; \mathrm{n}=104$ ], respectively).

Overall, patients treated with agalsidase alfa had a negligible annualized rate of change in LVMI (median [range] follow-up, 5.2 [1.5 to 11.4] years; Table 5). The mean (SEM) rate of LVMI change was 0.33 (0.10) $\mathrm{g} / \mathrm{m}^{2.7} / \mathrm{y}$ in males and $0.48(0.09)$ in females, compared with LVMI increasing at a rate of 4.07 (1.03) in untreated males and $2.31(0.81)$ in untreated females. In males and females with left ventricular hypertrophy $(\mathrm{LVH})$ at baseline, the differences in the rate of change between the ERT and untreated cohorts were suggestive of a treatment effect, although the SEMs for the untreated cohort values were high (Table 5). Six patients (all males, five with baseline LVH) were originally excluded from the FOS cohort because of prior renal dialysis and/or transplant; inclusion of these patients in the LVMI analysis had no significant impact on the findings (Supplemental Table 3).

A sensitivity analysis evaluating available 5-year follow-up LVMI data was conducted in 38 males and 40 females (median [range] treatment duration, 8.45 [0.9 to 11.7 ] years). In both, the rates of change in LVMI overall and in patients with baseline LVH were not significantly different from the corresponding values for the full FOS ERT cardiac cohort (Supplemental Table 4).

Treatment appeared to delay morbidity in patients with Fabry disease. After 24 months, the probability of a composite morbidity event was $16 \%$ (standard error [SE] 4.7\%) in the FOS ERT cohort overall compared with $~ 45 \%$ overall for the Banikazemi et al. [20] placebo group (Fig. 2a). Because the majority of patients (87\%) in the Banikazemi et al. placebo group were male, it is logical to compare the probability with that of the subset of male patients in the FOS Evaluable Treated Morbidity cohort, which was 26\% (SE 8.8\%). Given the more progressive nature of the disease in males, examining males only represents the worst case clinical scenario. Median (95\% CI) age at first event was also greater in agalsidase alfa-treated males and females, respectively, at $48(44.2,50.4)$ years and $56.9(54.1,59.9)$ years compared with untreated patients, at $\sim 41$ years and $\sim 53$ years, respectively (Fig. $2 \mathrm{~b}$ ).

Treatment with agalsidase alfa also appeared to have a positive effect on mortality. The estimated median survival time was 77.5 years for male patients from FOS compared with 60 years in patients without ERT [21] (Fig. 3). Sensitivity analyses excluding patients from FOS who initiated treatment later in life (i.e., aged $\geq 65$ years) or using random resampling of replicates to match the untreated population demographic yielded a median estimated $50 \%$ risk of mortality at age $\geq 70$ years.

Over $\sim 5$ years, treatment with agalsidase alfa slowed the progression of renal disease, particularly in males and in those with lower eGFR at baseline. Agalsidase alfa appeared to slow or stabilize progression of cardiomyopathy, including patients with LVH at baseline. Risk of morbidity was also reduced in treated patients from FOS and survival was improved. Our findings of beneficial effects of agalsidase alfa on the progression of renal impairment and cardiomyopathy outcomes concur broadly with those from previous analyses of treated patients from FOS and from other studies [11,13,15-17,24-26]. In treated patients from

Table 2

Ages and cardiovascular-related clinical characteristics at baseline of patients from FOS and Kampmann et al. [8] included in the analyses of the progression of cardiomyopathy.

\begin{tabular}{|c|c|c|c|c|}
\hline \multirow[b]{2}{*}{ Parameter (means) } & \multicolumn{2}{|c|}{ FOS Evaluable Treated Cardiac cohort $(\mathrm{n}=164)$} & \multicolumn{2}{|c|}{ Kampmann et al. [8] (untreated) $(\mathrm{n}=166)$} \\
\hline & Males $(\mathrm{n}=71)$ & Females $(\mathrm{n}=93)$ & Males $(\mathrm{n}=66)$ & Females $(\mathrm{n}=100)$ \\
\hline Age at baseline LVMI (yrs) & 31.3 & 41.9 & 33.8 & 38.0 \\
\hline BMI $\left(\mathrm{kg} / \mathrm{m}^{2}\right)$ & 21.9 & 24.4 & 21.7 & 23.5 \\
\hline $\operatorname{LVMI}\left(\mathrm{g} / \mathrm{m}^{2.7}\right)$ & 53.9 & 51.0 & 56.8 & 48.2 \\
\hline Systolic BP (mm Hg) & 124.7 & 124.3 & 126.5 & 126.1 \\
\hline Diastolic BP (mm Hg) & 73.4 & 70.5 & 71.8 & 71.7 \\
\hline Heart rate (beats/min) & 71.8 & 69.0 & 66.7 & 69.2 \\
\hline
\end{tabular}

BMI, body mass index; BP, blood pressure; FOS, Fabry Outcome Survey; LVMI, left ventricular mass indexed to height. 
Table 3

Two comparator patient cohorts (Banikazemi et al. [20] and Schiffmann et al. [21]) were utilized to evaluate morbidity and mortality outcomes for treated patients in two comparable FOS cohorts.

\begin{tabular}{|c|c|c|c|c|c|c|}
\hline Parameter (mean) & \multicolumn{3}{|c|}{$\begin{array}{l}\text { FOS Evaluable Treated } \\
\text { Morbidity cohort }\end{array}$} & \multicolumn{3}{|c|}{$\begin{array}{l}\text { Banikazemi et al. placebo } \\
\text { group [20] }\end{array}$} \\
\hline $\mathrm{n}$ & \multicolumn{3}{|c|}{79} & \multicolumn{3}{|l|}{31} \\
\hline Males (\%) & \multicolumn{3}{|c|}{48} & \multicolumn{3}{|l|}{87} \\
\hline Age at treatment start (yrs) & \multicolumn{3}{|c|}{51.6} & \multicolumn{3}{|c|}{44.3} \\
\hline Weight $(\mathrm{kg})$ & \multicolumn{3}{|c|}{71.4} & \multicolumn{3}{|l|}{70.2} \\
\hline Height $(\mathrm{cm})$ & \multicolumn{3}{|c|}{167.3} & \multicolumn{3}{|l|}{172.8} \\
\hline White, Asian (\%) & \multicolumn{3}{|c|}{80,18} & \multicolumn{3}{|l|}{87,3} \\
\hline Urine protein $(\mathrm{g} / 24 \mathrm{~h})$ & \multicolumn{3}{|c|}{0.6} & \multicolumn{3}{|l|}{1.1} \\
\hline Albumin/creatinine ratio $(\mathrm{mg} / \mathrm{g})$ & \multicolumn{3}{|c|}{910} & \multicolumn{3}{|l|}{900} \\
\hline Plasma creatinine $(\mathrm{mg} / \mathrm{dL})$ & \multicolumn{3}{|c|}{1.2} & \multicolumn{3}{|c|}{1.6} \\
\hline $\mathrm{eGFR}\left(\mathrm{mL} / \mathrm{min} / 1.73 \mathrm{~m}^{2}\right)$ & \multicolumn{3}{|c|}{63.9} & \multicolumn{3}{|c|}{52.4} \\
\hline Systolic BP (mm Hg) & \multicolumn{3}{|c|}{127} & \multicolumn{3}{|l|}{128} \\
\hline \multirow[t]{3}{*}{ Diastolic BP (mm Hg) } & \multicolumn{3}{|c|}{76} & \multicolumn{3}{|l|}{75} \\
\hline & \multicolumn{3}{|c|}{$\begin{array}{l}\text { FOS Evaluable Treated } \\
\text { cohort }\end{array}$} & \multicolumn{3}{|c|}{$\begin{array}{l}\text { Schiffmann et al. } \\
\text { (untreated) [21] }\end{array}$} \\
\hline & Males & Females & Total & Males & Females & Total \\
\hline $\mathrm{n}$ & 360 & 317 & 677 & 279 & 168 & 447 \\
\hline Age at data abstraction (yrs) & 40.8 & 50.6 & 45.4 & 38.6 & 44.9 & 41.0 \\
\hline Age at first symptom (yrs) & 14.3 & 24.9 & 19.0 & 10.5 & 17.4 & 12.6 \\
\hline Age at diagnosis (yrs) & 27.1 & 39.1 & 32.7 & 23.9 & 29.7 & 26.0 \\
\hline White (\%) & 82 & 84 & 83 & 85 & 86 & 85 \\
\hline
\end{tabular}

BP, blood pressure; eGFR, estimated glomerular filtration rate; FOS, Fabry Outcome Survey.

a This represents the ratio of albumin $(\mathrm{mg} / \mathrm{dL})$ to creatinine $(\mathrm{g} / \mathrm{dL})$ in urine.

the FOS with a mean follow-up period of 7.4 years, progression of renal impairment was stabilized in women and showed a small decline in men [16]. Furthermore, FOS 5-year data indicated a sustained reduction

Table 4

Progression of renal impairment determined as annualized rate of change in eGFR in male and female patients stratified by baseline eGFR and urinary protein levels.

\begin{tabular}{|c|c|c|c|c|}
\hline \multirow[b]{3}{*}{ Subgroup } & \multicolumn{2}{|c|}{$\begin{array}{l}\text { FOS Evaluable Treated } \\
\text { Renal cohort }\end{array}$} & \multicolumn{2}{|c|}{$\begin{array}{l}\text { Schiffmann et al. } \\
\text { (untreated) [21] }\end{array}$} \\
\hline & \multicolumn{4}{|c|}{ Mean annualized eGFR slope (SEM) [95\% CI], $\mathrm{mL} / \mathrm{min} / 1.73 \mathrm{~m}^{2} / \mathrm{y}$} \\
\hline & \multicolumn{2}{|l|}{$\mathrm{n}$} & \multicolumn{2}{|l|}{$\mathrm{n}$} \\
\hline \multicolumn{5}{|c|}{ Baseline eGFR category $\left(\mathrm{mL} / \mathrm{min} / 1.73 \mathrm{~m}^{2}\right)$} \\
\hline \multicolumn{5}{|l|}{ Males } \\
\hline$\geq 60$ & 117 & $\begin{array}{l}-1.68(0.19) \\
{[-2.05,-1.31]}\end{array}$ & 117 & $-3.0(0.1)$ \\
\hline$<60$ & 18 & $\begin{array}{l}-2.86(0.53) \\
{[-3.90,-1.83]}\end{array}$ & 28 & $-6.8(1.5)$ \\
\hline \multicolumn{5}{|l|}{ Females } \\
\hline$\geq 60$ & 111 & $\begin{array}{l}-0.43(0.21) \\
{[-0.83,-0.02]}\end{array}$ & 42 & $-0.9(0.9)$ \\
\hline$<60$ & 22 & $\begin{array}{l}0.36(0.42) \\
{[-0.47,1.19]}\end{array}$ & 13 & $-2.1(1.6)$ \\
\hline \multicolumn{5}{|c|}{ Baseline urinary protein level category $(\mathrm{g} / 24 \mathrm{~h})$} \\
\hline$\geq 1.0$ & 16 & $\begin{array}{l}-4.76(0.56) \\
{[-5.85,-3.66]}\end{array}$ & 22 & $-6.9(1.5)$ \\
\hline 0.1 to 1.0 & 74 & $\begin{array}{l}-1.62(0.23) \\
{[-2.08,-1.17]}\end{array}$ & 21 & $-3.3(1.8)$ \\
\hline$<0.1$ & 15 & $\begin{array}{l}-1.32(0.48) \\
{[-2.26,-0.38]}\end{array}$ & 18 & $-1.6(1.5)$ \\
\hline \multicolumn{5}{|l|}{ Females } \\
\hline$\geq 1.0$ & 17 & $\begin{array}{l}-0.41(0.51) \\
{[-1.42,0.59]}\end{array}$ & 5 & $-4.6(2.3)$ \\
\hline 0.1 to 1.0 & 70 & $\begin{array}{l}-0.44(0.26) \\
{[-0.95,0.07]}\end{array}$ & 17 & $-2.2(2.2)$ \\
\hline$<0.1$ & 22 & $\begin{array}{l}-0.16(0.42) \\
{[-0.97,0.66]}\end{array}$ & 7 & $-0.6(2.6)$ \\
\hline
\end{tabular}

$\mathrm{CI}$, confidence interval; eGFR, estimated glomerular filtration rate; FOS, Fabry Outcome Survey; SEM, standard error of the mean.

a Median (range) number of assessments for males and females: Schiffmann et al., 6 (3 to 33); FOS, 10 (3 to 70)
Table 5

Progression of cardiomyopathy, determined as annualized rate of change in LVMI, in male and female patients stratified by the presence or absence of LVH at baseline.

\begin{tabular}{|c|c|c|c|c|}
\hline \multirow[b]{3}{*}{ Baseline LVH status } & \multicolumn{2}{|c|}{$\begin{array}{l}\text { FOS Evaluable Treated } \\
\text { Cardiac cohort }\end{array}$} & \multicolumn{2}{|c|}{$\begin{array}{l}\text { Kampmann et al. } \\
\text { (untreated) [8] }\end{array}$} \\
\hline & \multicolumn{4}{|c|}{ Mean annualized LVMI slope (SEM) [95\% CI], ${ }^{\mathrm{a}} \mathrm{g} / \mathrm{m}^{2.7} / \mathrm{y}$} \\
\hline & $\mathrm{n}$ & & $\mathrm{n}$ & \\
\hline \multicolumn{5}{|l|}{ Males } \\
\hline Total & 71 & $\begin{array}{l}0.33(0.10) \\
{[0.13,0.53]}\end{array}$ & 39 & $4.07(1.03)$ \\
\hline LVH & 29 & $\begin{array}{l}0.19(0.16) \\
{[-0.13,0.50]}\end{array}$ & 18 & $6.59(8.5)$ \\
\hline No LVH & 42 & $\begin{array}{l}0.47(0.13) \\
{[0.22,0.72]}\end{array}$ & Not & \\
\hline \multicolumn{5}{|l|}{ Females } \\
\hline Total & 93 & $\begin{array}{l}0.48(0.09) \\
{[0.30,0.66]}\end{array}$ & 39 & $2.31(0.81)$ \\
\hline LVH & 45 & $\begin{array}{l}0.77(0.14) \\
{[0.49,1.05]}\end{array}$ & 15 & $3.77(7.7)$ \\
\hline No LVH & 48 & $\begin{array}{l}0.19(0.11) \\
{[-0.03,0.41]}\end{array}$ & Not & \\
\hline
\end{tabular}

CI, confidence interval; FOS, Fabry Outcome Survey; LVH, left ventricular hypertrophy; LVMI, left ventricular mass indexed to height; SEM, standard error of the mean.

a Median (range) follow-up period: FOS, 5.2 (1.5 to 11.4) years; Kampmann et al., 4.5 (1.1 to 9.0$)$ years [8].

b $95 \%$ CI for LVMI data were not published by Schiffmann et al. [21]

in LVMI in treated patients with baseline cardiac hypertrophy and stable LVMI in treated patients without baseline hypertrophy [11]. A randomized, double-blind clinical trial showed a significant reduction in left ventricular mass following 6 months of agalsidase alfa treatment compared with placebo [13]. One study of patients treated with either agalsidase alfa or beta for $\sim 5$ years suggests that the risk of a first or second renal, cardiac, or cerebrovascular event decreased with increasing treatment duration [27]. A recent cohort study of adults in England by Anderson et al. found that time on agalsidase alfa or agalsidase beta ERT was significantly associated with a reduction in LVMI as well as reduced risk of proteinuria and, among those without baseline proteinuria, a small increase in eGFR [28]. Another recent study by Juan et al. of six patients in Argentina with mild disease at baseline found that after 10 years of agalsidase beta ERT, no patients had a reduction in eGFR or progression to LVH [29]. Results of other studies suggest that long-term agalsidase beta delays the time to morbidity and death [10,20], although another study suggested no difference in event rate in patients with advanced disease at baseline versus an untreated control group [30].

The main strengths of our study are the inclusion of robust comparator datasets and the availability of data for $>700$ treated patients. Limitations include the fact that this was a retrospective analysis, not a randomized controlled clinical trial and, as discussed above, comparisons were not made with untreated patients from the same dataset. Matching at the patient level was not possible, as individual patient data for the untreated comparators was not available. Lack of randomization introduces the risk of bias in terms of treatment selection and ascertainment, with the possibility of more severely affected patients being more likely to be selected for ERT, to have more clinical attention, or to have sentinel events reported. The comparator arms, however, were the placebo arm from a randomized trial [20] or from retrospective [21] or cross-sectional [8] studies of untreated patients, some of whom later received ERT, except for patients from the latter study [8] who were included in the longitudinal study.

Using comparator groups from published literature presented a challenge in matching baseline demographic and clinical characteristics of the treated and untreated patient cohorts, the definitions of certain endpoints, and study duration and timing. Analysis cohorts from the FOS dataset were defined to best match the analysis populations in 
a FOS Evaluable Treated Morbidity Cohort $(\mathrm{n}=79,48 \%$ male $)$

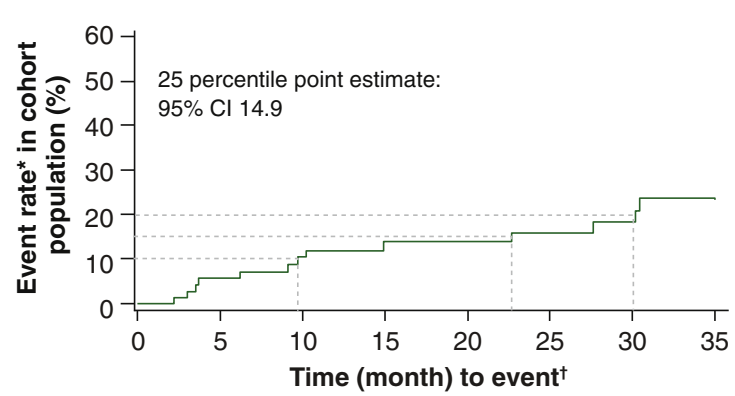

b

FOS Evaluable Treated Cohort

$(n=677)$

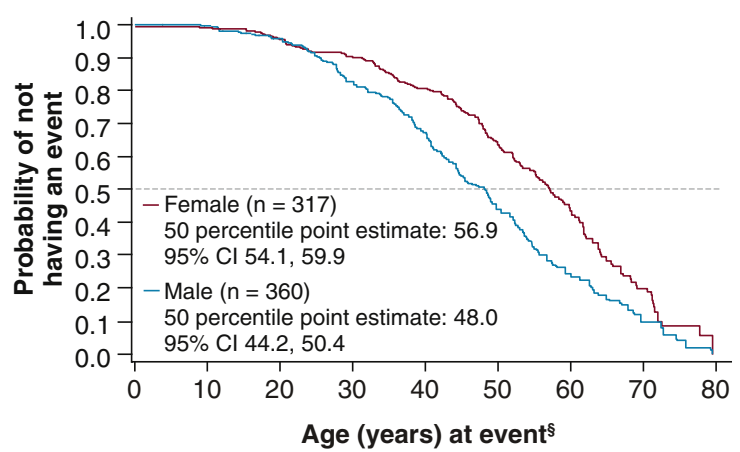

Banikazemi et al. Evaluable Populations (Untreated) [20] $(n=31,87 \%$ male $)$

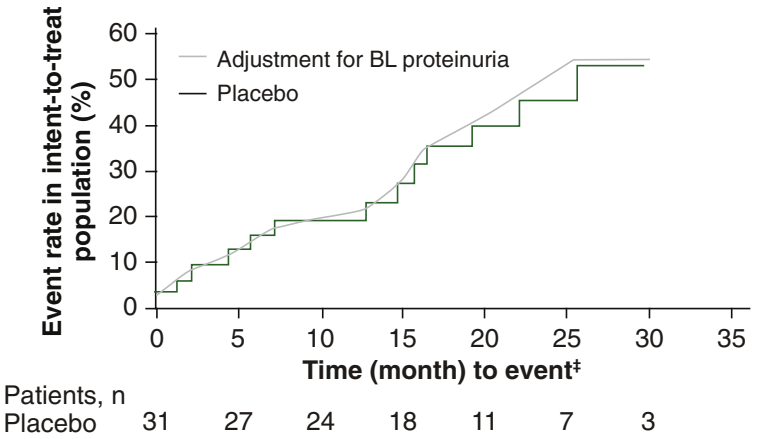

Schiffmann et al. Population (Untreated) [21] $(n=447)$

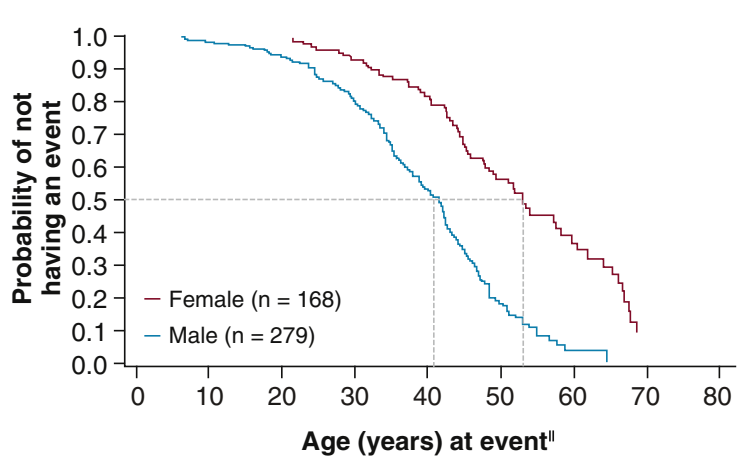

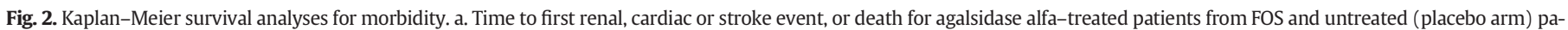

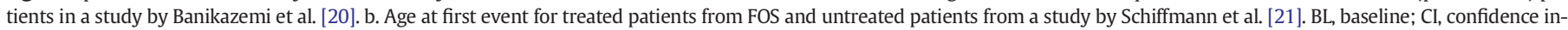

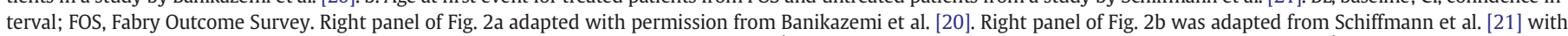

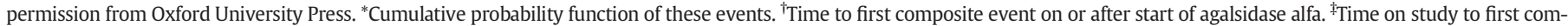
posite event. ${ }^{\S}$ Age at first composite event on or after start of agalsidase alfa. "Age at first composite event.

the published literature. This placed restrictions on which treated patients from FOS could be included in each comparator cohort. Hence, for some analyses, these groups were considerably smaller than the original FOS All Treated cohort. However, the percentage of patients with eGFR $<60 \mathrm{~mL} / \mathrm{min} / 1.73 \mathrm{~m}^{2}$ in the FOS Evaluable Treated Renal cohort is similar to that of the Schiffmann et al. population (15\% versus $21 \%$, respectively) and the percentage of patients with LVH in the FOS Evaluable Treated Cardiac cohort is similar to that of the Kampmann et al. population ( $45 \%$ versus $42 \%$, respectively). Additionally, to address differences in cases where the key baseline parameters of the FOS cohort did not match those of the published population, resampling simulations matching the baseline parameters were performed as sensitivity analyses. Another limitation of using data from the published literature was that further analyses, for example, of interactions between Fabry disease complications and outcomes, were not possible. Likewise, with no centralized reading of FOS echocardiograms, LVMI data from two different studies were compared. Patients who left FOS to be treated with another ERT were censored from the analyses from the date of leaving. This could be a source of bias because patients likely changed treatment due to intolerability or perceived lack of benefit; however, the use of Kaplan-Meier analyses should have reduced any impact of this on morbidity and survival findings. Finally, any long-term study introduces the potentially unavoidable risk that standards of diagnosis and care might change over time as well as availability of therapeutic agents, e.g., ACEI and ARB, a factor that may introduce bias and applied to all cohorts included in the analysis. Consideration of this risk is important, particularly in relation to the Schiffmann et al. [21] natural history study retrospective data, which was collected over several decades.

\section{Conclusions}

The findings from this retrospective comparison of data from FOS versus published studies support the long-term effectiveness of agalsidase alfa in the treatment of Fabry disease. The results show that treatment with agalsidase alfa slowed renal deterioration, slowed or stabilized progression of cardiomyopathy, and appeared to delay morbidity and death. Interpretation of these findings should take into account that they are based on retrospective comparisons with previously published data.

\section{Disclosure statement}

Michael Beck and Atul Mehta have received honoraria, travel support, and unrestricted grants from Actelion, BioMarin, Genzyme, and Shire. Derralynn Hughes has received travel and research grants and honoraria for speaking and consulting from Amicus, Genzyme/Sanofi, Protalix, and Shire. Guillem Pintos-Morell has received honoraria and travel support from Shire and travel support from BioMarin and Genzyme. Uma Ramaswami has received travel and research grants and honoraria for lectures from Genzyme and Shire. Christoph Kampmann has received honoraria for lectures and travel support from Actelion, BioMarin, Genzyme, Gore, and Shire. Anna Wijatyk is an employee of and owns shares in Shire, and Sylvain Larroque was an employee of Shire at the time this work was done. Roberto Giugliani has received consulting fees from Amicus, BioMarin, Genzyme, Shire, and Synageva; participated in clinical trials sponsored by Amicus, BioMarin, Genzyme, GlaxoSmithKline, Shire, and Synageva; and assisted in the design of and/or participated in clinical studies using 
a

FOS Evaluable Treated Cohort

$(n=677)$

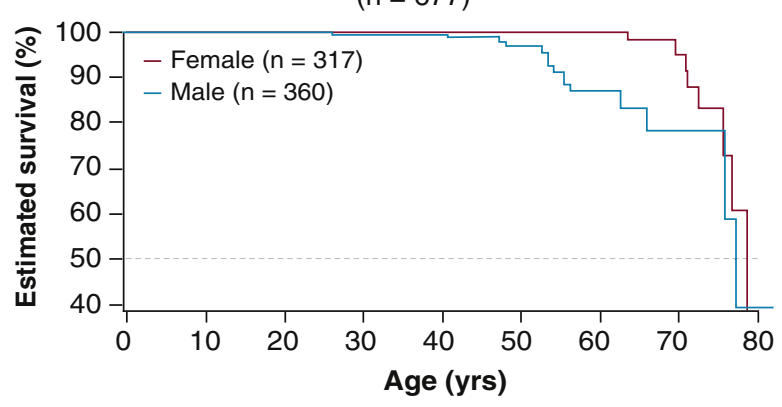

b Schiffmann et al. Untreated Population [21]

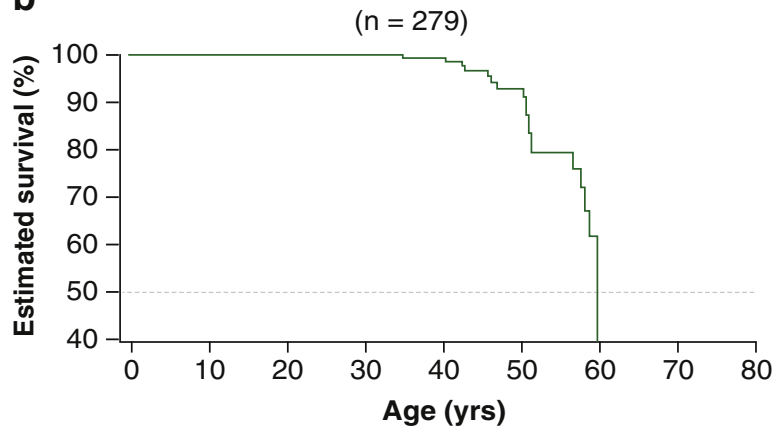

Fig. 3. Kaplan-Meier survival analyses (mortality) in a. treated patients from FOS and b. untreated male patients from Schiffmann et al. [21]. FOS, Fabry Outcome Survey.

Fig. 3b was adapted from Schiffmann et al. [21] with permission from Oxford University Press.

products manufactured by Actelion, BioMarin, Genzyme, and Shire. Michael West has received research funds, consulting fees, speaker fees, and/or travel support from Actelion, Amicus, Excelsior, Genzyme, GlaxoSmithKline, Shire, Sumitomo, and Synageva.

\section{Role of the funding source}

Shire participated in the study design; collection, analysis, and interpretation of data; funded medical writing support provided by Annie Rowe and Margit Rezabek of Excel Scientific Solutions; and reviewed the manuscript for scientific accuracy.

\section{Acknowledgments}

The Fabry Outcome Survey (FOS) is supported by Shire. We thank the patients who participated in FOS.

\section{Appendix A. Supplementary data}

Supplementary data to this article can be found online at http://dx. doi.org/10.1016/j.ymgmr.2015.02.002.

\section{References}

[1] S.C. Garman, D.N. Garboczi, The molecular defect leading to Fabry disease: structure of human alpha-galactosidase, J. Mol. Biol. 337 (2004) 319-335.

[2] A. Mehta, J.T. Clarke, R. Giugliani, et al., Natural course of Fabry disease: changing pattern of causes of death in FOS - Fabry Outcome Survey, J. Med. Genet. 46 (2009) 548-552.
[3] K.D. MacDermot, A. Holmes, A.H. Miners, Anderson-Fabry disease: clinical manifestations and impact of disease in a cohort of 60 obligate carrier females, J. Med. Genet. 38 (2001) 769-775.

[4] K.D. MacDermot, A. Holmes, A.H. Miners, Natural history of Fabry disease in affected males and obligate carrier females, J. Inherit. Metab. Dis. 24 (Suppl. 2) (2001) 13-14 (discussion 11-12).

[5] M. West, K. Nicholls, A. Mehta, et al., Agalsidase alfa and kidney dysfunction in Fabry disease, J. Am. Soc. Nephrol. 20 (2009) 1132-1139.

[6] K. Sims, J. Politei, M. Banikazemi, P. Lee, Stroke in Fabry disease frequently occurs before diagnosis and in the absence of other clinical events: natural history data from the Fabry Registry, Stroke 40 (2009) 788-794.

[7] M. Branton, R. Schiffmann, J.B. Kopp, Natural history and treatment of renal involvement in Fabry disease, J. Am. Soc. Nephrol. 13 (Suppl. 2) (2002) S139-S143.

[8] C. Kampmann, A. Linhart, F. Baehner, et al., Onset and progression of the AndersonFabry disease related cardiomyopathy, Int. J. Cardiol. 130 (2008) 367-373.

[9] R. Schiffmann, J.B. Kopp, H.A. Austin III, et al., Enzyme replacement therapy in Fabry disease: a randomized controlled trial, JAMA 285 (2001) 2743-2749.

[10] F. Breunig, F. Weidemann, J. Strotmann, A. Knoll, C. Wanner, Clinical benefit of enzyme replacement therapy in Fabry disease, Kidney Int. 69 (2006) 1216-1221.

[11] A. Mehta, M. Beck, P. Elliott, et al., Enzyme replacement therapy with agalsidase alfa in patients with Fabry's disease: an analysis of registry data, Lancet 374 (2009) 1986-1996.

[12] R.P. El Dib, P. Nascimento, G.M. Pastores, Enzyme replacement therapy for Anderson-Fabry disease, Cochrane Database Syst. Rev. 2 (2013) CD006663.

[13] D.A. Hughes, P.M. Elliott, J. Shah, et al., Effects of enzyme replacement therapy on the cardiomyopathy of Anderson-Fabry disease: a randomised, double-blind, placebocontrolled clinical trial of agalsidase alfa, Heart 94 (2008) 153-158.

[14] E. Hernberg-Stahl, Organization and technical aspects of FOS - the Fabry Outcome Survey, in: A. Mehta, M. Beck, G. Sunder-Plassmann (Eds.), Fabry Disease: Perspectives From 5 Years of FOS. Oxford PharmaGenesis, Oxford, 2006.

[15] M. Beck, R. Ricci, U. Widmer, et al., Fabry disease: overall effects of agalsidase alfa treatment, Eur. J. Clin. Investig. 34 (2004) 838-844.

[16] S. Feriozzi, J. Torras, M. Cybulla, K. Nicholls, G. Sunder-Plassmann, M. West, The effectiveness of long-term agalsidase alfa therapy in the treatment of Fabry nephropathy, Clin. J. Am. Soc. Nephrol. 7 (2012) 60-69.

[17] A. Schwarting, F. Dehout, S. Feriozzi, M. Beck, A. Mehta, G. Sunder-Plassmann, Enzyme replacement therapy and renal function in 201 patients with Fabry disease, Clin. Nephrol. 66 (2006) 77-74.

[18] A. Linhart, C. Kampmann, J.L. Zamorano, et al., Cardiac manifestations of AndersonFabry disease: results from the international Fabry outcome survey, Eur. Heart J. 28 (2007) 1228-1235

[19] A. Mehta, R. Ricci, U. Widmer, et al., Fabry disease defined: baseline clinical manifestations of 366 patients in the Fabry Outcome Survey, Eur. J. Clin. Investig. 34 (2004) 236-242.

[20] M. Banikazemi, J. Bultas, S. Waldek, et al., Agalsidase-beta therapy for advanced Fabry disease: a randomized trial, Ann. Intern. Med. 146 (2007) 77-86.

[21] R. Schiffmann, D.G. Warnock, M. Banikazemi, et al., Fabry disease: progression of nephropathy, and prevalence of cardiac and cerebrovascular events before enzyme replacement therapy, Nephrol. Dial. Transplant. 24 (2009) 2102-2111.

[22] K/DOQI clinical practice guidelines for chronic kidney disease: evaluation, classification, and stratification, Am. J. Kidney Dis. 39 (2002) S1-S266.

[23] R.B. Devereux, M.J. Koren, G. de Simone, M.J. Roman, J.H. Laragh, Left ventricular mass as a measure of preclinical hypertensive disease, Am. J. Hypertens. 5 (1992) 175S-181S.

[24] R. Schiffmann, M. Ries, M. Timmons, J.T. Flaherty, R.O. Brady, Long-term therapy with agalsidase alfa for Fabry disease: safety and effects on renal function in a home infusion setting, Nephrol. Dial. Transplant. 21 (2006) 345-354.

[25] C. Kampmann, A. Linhart, R.B. Devereux, R. Schiffmann, Effect of agalsidase alfa replacement therapy on Fabry disease-related hypertrophic cardiomyopathy: a 12- to 36-month, retrospective, blinded echocardiographic pooled analysis, Clin. Ther. 31 (2009) 1966-1976.

[26] J. Zamorano, V. Serra, L. Perez de Isla, et al., Usefulness of tissue Doppler on early detection of cardiac disease in Fabry patients and potential role of enzyme replacement therapy (ERT) for avoiding progression of disease, Eur. J. Echocardiogr. 12 (2011) 671-677.

[27] S.M. Rombach, B.E. Smid, M.G. Bouwman, G.E. Linthorst, M.G. Dijkgraaf, C.E. Hollak, Long term enzyme replacement therapy for Fabry disease: effectiveness on kidney, heart and brain, Orphanet J. Rare Dis. 8 (2013) 47.

[28] L.J. Anderson, K.M. Wyatt, W. Henley, et al., Long-term effectiveness of enzyme replacement therapy in Fabry disease: results from the NCS-LSD cohort study, J. Inherit. Metab. Dis. 37 (2014) 969-978.

[29] J. Politei, H. Amartino, A.B. Schenone, et al., Fabry disease: multidisciplinary evaluation after 10 years of treatment with agalsidase beta, JIMD Rep. 16 (2014) 7-14.

[30] F. Weidemann, M. Niemann, S. Stork, et al., Long-term outcome of enzymereplacement therapy in advanced Fabry disease: evidence for disease progression towards serious complications, J. Intern. Med. 274 (2013) 331-341. 